\title{
Geographic Information System Oriented GIS Automatic Surveying and Mapping Technology
}

\author{
Yang Qianqian ${ }^{1}$, Qin Hui ${ }^{2}$, Chen Tianwei ${ }^{1}$ \\ ${ }^{1}$ College of Geomatics and Geoinformation, Guilin University of Technology, Guilin, Guangxi, 541004 \\ ${ }^{2}$ Guangdong Academy of scientific and technological cadres, Guangdong Zhuhai, 519090
}

Keywords: geographic information system; surveying and mapping; extended application

\begin{abstract}
With the growth of the economy, a higher and more urgent requirement for the pioneers of the development of the national economy is put forward, and the traditional means of surveying and mapping cannot meet the requirements of the accuracy and efficiency of the surveying and mapping. The wide application of geographic information system (GIS) in surveying and mapping greatly improves the efficiency of surveying and mapping and the reality of data. This article mainly analyzes and discusses the application of GIS in surveying and mapping field, providing reference for improving the accuracy and efficiency of surveying and mapping.
\end{abstract}

\section{Introduction}

Geographic Information System (GIS) can be simply defined as a computer system that collects, stores, manages, analyzes, displays and applies geographic information. GIS is the product of geography and information technology. It provides new technology and methods for solving various problems related to spatial location. With the development of nearly 50 years [1], with the development of computer technology, space technology and modern information infrastructure, geographic information system (GIS) has been widely used in various fields, and has produced great economic and social benefits. The work of GIS in China started slightly later than the international level, but its development is fast and the situation is gratifying. In the early 1970s, our country began to popularize the application of computers in surveying, mapping and remote sensing [2]. The demand for environmental remote sensing resource survey and the development of aerial photogrammetry and topographic mapping laid a good foundation for the development of GIS. From 1980s, the first Institute of geographic information system (GIS) of the National Institute of remote sensing applications of the Chinese Academy of Sciences (1980) marked the beginning of the work of China's geographic information system. Since 1990s, GIS has entered a stage of rapid development. From the preliminary development period of the research experiment and local application to the practical and productive, the geographic information system provides the analysis and decision basis for the major problems of the national economy. After nearly 40 years of development, China's geographic information system has made significant progress [3-5]. The research and application of geographic information system has gone to industrialization.

\section{Automatic Surveying and Mapping Technology}

\subsection{Gps Technology}

GPS technology has the ability of all-around, real-time 3D navigation and location in sea, land and air. It's a new generation of satellite navigation and positioning system, multi-function mapping tool. Compared with the traditional measurement and location, it has the characteristics of good anti-interference, confidentiality, multi functions, global, full coverage, all-weather and high accuracy. Especially, the positioning accuracy of RTK can reach centimeter level, and it has been widely applied in water location. 


\subsection{GPS Technology}

Technical RS technology does not directly contact the object studied, and the characteristic information is generally the reflection, radiation and emission of electromagnetic waves. Through transmission and processing, the information that people are interested in, such as satellite, aeronautical, aerospace photogrammetry and so on, is extracted from it, and it helps the surveying and mapping personnel to obtain the surveying ground by stereoscopic photography. Three dimensional information makes people understand the characteristics of Surveying and mapping more intuitively.

\section{GIS Automatic Surveying and Mapping Technology}

In the rapid pace of economic and technological development, our technology, products, ideas must be improved with the development of the times, or the immature technology will be eliminated by this era. In order to adapt to the rapid development of modern surveying and mapping technology automation technology, terrain mapping has to get rid of the traditional model, the pursuit of more efficient and accurate, for all kinds of planning and construction provides unparalleled role. The traditional surveying and mapping technology has various drawbacks, and the modern mapping technology automation technology has brought the fresh blood into the modern topographic surveying and mapping, which has brought great promotion effect, which is mainly shown in the following aspects:

We know that the traditional surveying and mapping before the use of the original mapping equipment, tedious surveying and mapping process to increase manpower, in the surveying and mapping after the artificial drawing of the corresponding graphics, very troublesome. With the development of science and technology, many advanced and convenient tools with large quantity of science and technology are extended, and the computer is used to replace manpower. On the one hand, the input of manpower is reduced on the other hand and the error rate is reduced. Surveying and mapping personnel can complete the work of Surveying and mapping in the office by manipulating the computer. At the same time, modern surveying and mapping technology can also automatically generate graphics by related technology to the topography of the topographic map, thus saving the mapping of Surveying and mapping personnel. Modern surveying and mapping technology makes topography more simple and fast.

We know that topographic surveying is by measuring the related terrain and drawing relevant graphics through the data that is effectively measured. It is the use of regional planning, strategy setting, application to geography teaching and editing, so as to retain relevant geographic information for the country and transform it into various industries in the country. Therefore, the accuracy of terrain mapping requires a certain degree of accuracy to meet the above requirements. Traditional surveying and mapping technology is purely manual measurement and calculation, so it is easy to error, without high accuracy and it is difficult to detect and change after the error. Modern surveying and mapping technology can effectively solve this problem. It is more accurate to measure the terrain in need of Surveying and automatically draw the corresponding topographic map through precise measuring instruments and intelligent drawing means. Modern electronic map makes use of intelligent mapping technology to make us more intuitive and accurate. . Therefore, modern surveying and mapping technology automation technology makes terrain mapping more accurate.

Traditional surveying and mapping technology requires workers to bring instruments to all kinds of terrain, such as steep mountains, complex wetlands, and turbulent rivers. In a dangerous environment, a lot of uncertain factors and unsafe factors are added, which pose a threat to human life. The application of modern surveying and mapping technology in topographic surveying and mapping has solved this problem, which not only reduces the work intensity of the surveying and mapping workers, but also increases the safety and security of the staff. Modern technology makes workers do not have to map in dangerous terrain, can work through modern technology or satellite technology, improve work efficiency and increase the safety factor. 


\section{The application of $\mathbf{4}$ geographic information system in surveying and mapping}

\subsection{Data Source}

Data is very important for surveying and mapping. Data collection is an important function of GIS. From the type of data sources, geographic information system (GIS) data can be roughly divided into original data (first hand data) or processed data (secondhand data). In addition, data sources can be divided into two types of non electronic data and electronic data.

First hand data is the data that is collected directly. The traditional field data acquisition tools include three feet, a ruler, a compass, a flatmeter, a gradient instrument, a ruler, and so on. The results of data acquisition often need to be recorded on the paper, and the geographic information system (GIS) uses a modern measuring instrument represented by the global positioning instrument, laser rangefinder and total station instrument. Some instruments are gradually replacing these traditional data acquisition methods. The global positioning instrument, laser range finder, total station and so on can directly connect with the data recorder, and store the measured location, distance and azimuth data in the data recorder, and can also be stored directly on the hard disk of the portable computer, which greatly simplifies the acquisition of the first hand data. After interpreting, editing and processing the first hand data, it becomes secondhand data. Most of the data in geographic information system (GIS) are secondhand data, which mainly include map, statistical data report and image map.

\subsection{Data Collection}

According to the needs of Surveying and mapping tasks, the data of various data sources are converted into the format of the geographic information system (GIS) software which can be identified and used as data acquisition. Map Digitalization is one of the data acquisition methods adopted by many geographic information system (GIS) systems. For large surveying and mapping projects, the modern geographic information system (GIS) technology can automate the process by scanning technology. For smaller surveying and mapping projects, it needs to be digitalized manually (for example, using digital board). Attribute data is an important part of geographic data in geographic information system (GIS). It can input attribute data directly through keyboard. In geographic information system (GIS), attribute data is usually managed by professional data software, including collection of attribute data. In order to make full use of the existing data resources of various digital forms, transferring or transferring attribute data from other database systems will be a very important and necessary means to obtain the attribute data of geographic information system (GIS).

We know that geographic information system (GIS) usually classifies and manages the geographical elements needed for drawing, and the classification of elements is usually embodied in the classification and coding of attribute data. Classification and coding of attribute data are also very helpful for data exchange and sharing.

\subsection{Data Editing and Processing}

The geographical data after drawing collection often needs to be edited and processed, this is because: one, the possible errors in the data; two, to facilitate the query and analysis of the geographic information system (GIS), the data need to be further processed, such as the generation of topological relations and new attribute data, and three, some data. It is dynamic and needs to be updated continuously; four, for some special applications or data from other systems, it is possible to convert data format. Modern geographic information system (GIS) technology provides many tools to edit and process geographic data.

\subsection{Space Data Management}

Geographic Information System (GIS) software has multiple ways to manage spatial data. The early geographic information system (GIS) is based on files to manage spatial data, and is characterized by the development and design of different format file systems for different data models. This management mode is flexible, so it is still in use. The drawback is that it cannot 
manage huge amounts of data, and the query and management function for attributes is much weaker than professional DBMS.

For small geographic information system (GIS) projects, storing geographic information in simple files is enough. However, when data is large and multiple users use data at the same time, it is best to use a database management system (DBMS) to help store, organize, and manage spatial data.

\subsection{Spatial Data Analysis}

After the preprocessing of the previous four stages of mapping and mapping, the geographic information system (GIS) carries out spatial analysis and calculation of the preprocessed graphic data, and describes and studies space objects quantitatively and studies by spatial location and spatial objects. Spatial analysis function is the most complex and most important function of geographic information system (GIS). The function of spatial analysis is combined with many disciplines, such as geophysics, geography, regional science, and so on. Space statistics and topology should be used in the analysis and description of space composition. At present, spatial analysis technology is not fully mature, the main spatial analysis methods are mainly spatial simulation analysis, spatial simulation analysis is mainly through specialized model of spatial entities and relationships to be transformed and abstracted, for the further analysis of the operation of the system.

\section{Conclusion}

In order to make a better quality of life in continuous exploration, research, we use machinery instead of human resources, using software instead of the brain, so the traditional mapping technology to $3 \mathrm{G}$ technology, digital photogrammetry and artificial intelligence development is an inevitable trend.

\section{Acknowledgement}

Funding for the Key Laboratory of space information and mapping in Guangxi (15-140-07-09), Guangxi Natural Science Foundation Project (number: 2017GXNSFAA198308)

\section{References}

[1] Hesse W, Hazelton W, Williamson I P. Object-oriented concepts for software development and conceptual modelling in GIS and surveying[J]. Object-Oriented Programming Systems, 2005.

[2] Suryawanshl S L, Bhutada S H, Bombale V T, et al. Automatic generation of land capability map using remote sensing and geographic information system techniques. [J]. International Journal of Agricultural Engineering, 2010.

[3] Bazemore A, Phillips R L, Miyoshi T. Harnessing Geographic Information Systems (GIS) to enable community-oriented primary care. [J]. Journal of the American Board of Family Medicine Jabfm, 2010, 23(1):22.

[4] Martino S D, Ferrucci F, Paolino L, et al. Towards the automatic generation of web GIS[C]// ACM International Symposium on Advances in Geographic Information Systems. ACM, 2007:57.

[5] Xiaodan M, Wang Y, Ma Junhai|, et al. Research and practice on teaching system of GIS in surveying and mapping engineering based on talented plan[J]. Engineering of Surveying \& Mapping, 2013, 22(5):93-96. 